\title{
Novel allosteric mechanism of p53 activation by small molecules for targeted anticancer
}

\section{therapy}

Joanna Zawacka-Pankau ${ }^{1, \&}$, Vera V. Grinkevich ${ }^{1}$, Mikhail Burmakin ${ }^{1}$, Aparna Vema ${ }^{2, \#}$, Karin Ridderstråle ${ }^{1, \#}$, Natalia Issaeva ${ }^{3}$, Virginia Andreotti ${ }^{4}$, Eleanor R. Dickinson ${ }^{5}$, Elisabeth Hedström $^{1}$, Clemens Spinnler ${ }^{1}$, Alberto Inga ${ }^{6}$, Lars-Gunnar Larsson $^{1}$, Anders Karlén ${ }^{3}$, Olga Tarasova $^{7}$, Vladimir Poroikov $^{7}$, Sergey Lavrenov ${ }^{7}$, Maria Preobrazhenskaya $^{8}$, Margareta Wilhelm ${ }^{1}$, Perdita E. Barran ${ }^{5}$, Andrei L. Okorokov ${ }^{9}$, and Galina Selivanova ${ }^{1, \&}$

${ }^{1}$ Department of Microbiology, Tumor and Cell Biology, Karolinska Institute, Solnavägen 9, Stockholm, SE 171 65, Sweden

${ }^{2}$ Division of Organic Pharmaceutical Chemistry, Department of Medicinal Chemistry, Uppsala University, P.O. Box 574, BMC, S-751 23 Uppsala, Sweden

${ }^{3}$ Department of Otolaryngology, Yale Physicians Building, 800 Howard Ave, 4th Fl, New Haven, CT 06519

${ }^{4}$ Unit of Molecular Mutagenesis and DNA repair, National Institute for Cancer Research, IST, L.go R. Benzi X, 16132 Genoa, Italy

${ }^{5}$ Manchester Institute of Biotechnology, The School of Chemistry, The University of Manchester, 131 Princess Street Manchester, M1 7DN, UK

${ }^{6}$ Centre for Integrative Biology, CIBIO, University of Trento, via Sommarive 9, 38123, Trento, Italy

${ }^{7}$ Institute of Biomedical Chemistry, Russian Academy of Medical Sciences, 119121, Moscow Russia

${ }^{8}$ Gausse Institute of novel antibiotics, Russian Academy of medical sciences, 119021, Moscow, Russia

${ }^{10}$ Wolfson Institute for Biomedical Research, University College London, Gower Street, London, WC1E 6BT, UK

frame - the author has deceased during the course of the project

\& shared senior authorship and correspondence:

Galina Selivanova, Galina.Selivanova@ki.se, +46852486302, fax: +46 8342651

Joanna Zawacka-Pankau, joannazawackapankau1 @ gmail.com

\# - these authors contributed equally to this study

Running title: Allosteric inhibition of p53/MDM2 and p53/MDMX

Key words: p53; small molecules; allosteric inhibition; MDM2; MDMX 


\begin{abstract}
Given the immense significance of p53 restoration for anti-cancer therapy and that p53activating molecules are in clinical trials, elucidation of the mechanisms of action of p53activating molecules is of the utmost importance. Here we report a discovery of a novel allosteric modulation of p53 by small molecules, which is an unexpected turn in the p53 story. We identified a structural element involved in allosteric regulation of p53, whose targeting by small molecules RITA, PpIX and licofelone blocks the binding of two p53 inhibitors, MDM2 and MDMX, thereby restoring p53 function. Deletion and mutation analysis followed by molecular modeling and its thorough validation, identified the key p53 residues S33 and S37 targeted by RITA and PpIX. We propose that the binding of small molecules to the identified site in p53 induces a conformational trap preventing p53 from the interaction with MDM2 and MDMX. These results point to a high potential of allosteric activators as targeted drugs. Our study provides a basis for the development of therapeutics with a novel mechanism of action, thus extending the p53 pharmacopeia.
\end{abstract}




\section{Introduction}

Half of human tumors express inactive mutant p53, while wild type p53 is rendered functionally inert in cancer mainly due to the deregulated E3 ubiquitin ligase MDM2 and its homolog MDMX, which together potently inhibit p53 (Vousden KH et al. 2009). Several molecules targeting the p53-binding pocket of MDM2, such as nutlin and MI compounds, or the inhibitors of E3 ubiqutin ligase activity of MDM2, have been shown to induce p53-dependent growth suppression (Vassilev et al. 2004; Shangary et al. 2008; Yang et al., 2005) and some are currently being tested in clinical trials (Hoe et al. 2014). However, MI compounds and nutlins cannot ablate another key p53 inhibitor - MDMX, which makes them less efficient in tumors overexpressing MDMX (Toledo et al. 2007).

We have identified a small molecule RITA in cell-based screen for p53 reactivating compounds (Issaeva et al. 2004). RITA restores wild type p53 in tumor cells by preventing p53/MDM2 interaction (Issaeva et al. 2004; Enge et al. 2009; Grinkevich et al. 2009). Next we have found, that protoporphyrin IX (PpIX), a metabolite of aminolevulinic acid, a pro-drug applied in photodynamic therapy of cancer, activates p53 by inhibition of p53/MDM2 complex (Zawacka-Pankau et al. 2007). In contrast to nutlin, RITA does not target MDM2, but binds to the p53 N-terminus (Issaeva et al. 2004). However, how the binding to 553 affects p53/MDM2 complex remains unclear.

In the present study, we applied state-of-the art molecular and cell biology approaches and molecular modeling to map the region within p53 targeted by small molecules and to address the mechanism of their action. We found that RITA targets p53 outside of the MDM2-binding locus and identified the key structural elements in RITA molecule along with contact residues in $\mathrm{p} 53$, which are critical for the interaction. We found that the binding of RITA promotes a compact conformation of partially unstructured Nterminus, which inhibits the interaction with MDM2 and MDMX. Further, another p53 
activator PpIX, acts through the similar mechanism. Based on our results, we propose a model of a new allosteric mechanism of p53 activation. Using our model and chemoinformatic approaches, we have identified licofelone, dual COX/LOX inhibitor, which blocks p53/MDM2 interaction via the mechanism that we uncovered.

\section{Results}

RITA selectively interacts with p53 in cancer cells.

Our previous findings indicate that RITA interacts with N-terminal region of p53 in vitro (Issaeva et al. 2004). To test whether RITA targets p53 in a cellular context, we analyzed $\left[{ }^{14} \mathrm{C}\right]$-RITA complexes with proteins formed in HCT 116 colon carcinoma cells carrying wild-type p53 and in their p53-null counterparts (HCT 116 TPp53-/-). To visualize the complexes, we electrophoretically separated them and detected the position of RITA and p53 by autoradiography and Western blot, respectively. Under mild denaturing conditions, $\left[{ }^{14} \mathrm{C}\right]$-RITA migrated with the electrophoretic front in the lysates of HCT 116 TPp53-/(Figure 1A), whereas in the lysates of cells HCT 116 its migration was shifted, indicating the formation of complexes. The position of the major band coincided with that of p53. Further, immunodepletion of p53 from the lysates (Figure 1A) significantly decreased its intensity supporting the notion that it represents p53/RITA complex.

Next, we developed a small-molecule band shift assay, analogous to the commonly used band shift assay for detection of protein/DNA binding. We separated $\left[{ }^{14} \mathrm{C}\right]-$ RITA/cellular protein complexes by native electrophoresis and detected $\left[{ }^{14} \mathrm{C}\right]$-RITA by autoradiography (Figure 1B). The position of RITA/protein complex coincided with that of p53 (Figure 1B). The absence of similar pattern in p53-null cells (Figure 1B) indicates that it 
represents RITA bound to $\mathrm{p} 53$. Taken together, our data provides strong evidence for the selective interaction of RITA with p53 in cells.

Interaction of RITA with the N-terminus of p53

Next, we analyzed the interaction of RITA with the recombinant p53 Nterminus employing our small-molecule band shift assay. Upon incubation ${ }^{14} \mathrm{C}$-RITA formed a complex with Gluthathione-S-transferase (GST)-fusion p53 N-terminus (Np53) (2-65), as manifested by the co-migration with Np53 (Figure 1C). In contrast, RITA did not associate with human fibrinogen (Figure 1C), suggesting a selective interaction with p53. Human serum albumin (HSA), known blood carrier of various drugs (Koehler et al. 2002), served as binding control (Figure 1C). Under denaturating conditions $\left[{ }^{14} \mathrm{C}\right]$-RITA/protein complexes were disrupted (Figure 1D), suggesting that this interaction is reversible.

Non-labeled RITA readily competed out the $\left[{ }^{14} \mathrm{C}\right]$-RITA from the complex with Np53 at a low molecular excess, 1:1 or 1:2.5 (Figure S1A). However, it did not efficiently compete with the $\left[{ }^{14} \mathrm{C}\right]-\mathrm{RITA} / \mathrm{HSA}$ complex (Figure S1B) suggesting a different mode of interaction.

\section{Mapping RITA binding site using deletion and point mutants}

To identify key p53 residues involved in binding to RITA, we generated a series of p53 deletion mutants and assessed their interaction with RITA (Figure 2A).

Deletion of first 25 residues containing MDM2 binding site or mutations in p53 residues 22/23 required for MDM2 interaction did not alter p53 binding to RITA (Figure 2B). These results, as well as a weak, if any, evidence of interaction with Np53(2-25) (Figure 2B, upper panel) argue against the binding of RITA within the MDM2 site of p53. 
Notably, Np53(38-53) was not interacting with RITA either (Figure 2B, lower panel). Together, our results suggest that RITA target sequence is located between residues 25-38 (Figure 2A and B). Further analysis revealed that Np53(35-65) interacted with RITA approximately 50\% less efficiently than Np53(2-65) (Figure 2B). Therefore, we concluded that RITA targets residues located in the proximity to leucine 35.

\section{Molecular modeling of RITA/p53 complex}

Based on the available information on the structural organization of the p53 Nterminus (Kussie et al., 1996; Okorokov et al., 2006; Lowry et al., 2008; Espinoza-Fonesca 2009) and our deletion analysis, we carried out Monte Carlo conformational search to explore the possible binding modes of RITA to the p53 N-terminus (MacroModel, 2008). The MCMM-LMOD search on the RITA-p53 complex found 3492 low energy binding modes within $5 \mathrm{kcal} / \mathrm{mol}$ above the global minimum. Among these the tenth lowest energy binding mode, $2.1 \mathrm{kcal} / \mathrm{mol}$ above the global minimum, appeared reasonable with respect to the placement and orientation of RITA.

This model implies that the binding of RITA involves the formation of hydrogen bonds between its terminal hydroxyl groups and serine 33 and serine 37 of p53, as well as hydrophobic interactions with proline 34 and 36 via one of its thiophene and the furan rings (Figure 3A and B and Supplemental video 1). Hydrogen bonds and hydrophobic interactions between RITA and p53 SPLPS amino acid sequence result in a limited flexibility of this region (Figure $\mathbf{3 A}$ and $\mathbf{B}$ ).

Molecular dynamic simulations suggest that leucine-rich hydrophobic clusters within residues 19-26 and 32-37 stabilize the folding and formation of $\alpha$-helixes in the Nterminus (Espinoza-Fonesca et al. 2009). According to this study, MDM2-contacting residues F19, W23 and L26 within the $\alpha$-helix of p53 (residues 16-26) are facing inwards and are 
tacked inside, stabilized by the formation of hydrophobic leucine clusters, while more hydrophilic residues of the $\alpha$-helix are exposed to the solvent. This is supported by the tryptophan fluorescence assay, which demonstrated that W23 is shielded from the solvent (Kar et al., 2002). On the other hand, the X-ray structure of the MDM2-p53 peptide complex (1YCQ.pdb) shows that MDM2-contacting residues are facing out (Figure 3C). This indicates that the binding to MDM2 requires a partial unwinding of the $\alpha$-helix to flex out F19, W23 and L26, as illustrated in Figure 3B and 3C. This notion has been further corroborated by recent studies which revealed that segments $23-31$ and $31-53$ of the p53 Nterminus can exert remote effects on its flexibility upon the phosphorylation of residues S33, S46 and T81, as well as upon MDM2 binding (Lum et al., 2012).

Our model indicates that the binding of RITA increases the rigidity of the prolinecontaining SPLPS motif. This produces a conformational trap, which limits the flexibility of the amphipathic $\alpha$-helix. We propose that constraints imposed by RITA prevent 'flipping out' of F19, W23 and L26, thus counteracting the p53/MDM2 interaction (Figure 3B and 3C).

Conformational change induced by RITA is expected to impinge on other protein interactions involving the p53 N-terminus. The binding of p53 to the MDM2 homolog, MDMX requires the formation of an $\alpha$-helix as well as exposure of the same p53 residues, as facilitated by MDM2. We therefore reasoned that the conformational change induced by RITA might in addition abrogate the binding of MDMX as well.

RITA ablates p53/MDMX interaction in cells and in vitro.

To assess whether RITA could inhibit p53/MDMX complex, we performed coimmunoprecipitation experiments. As shown in Figure 3D, RITA triggered pronounced reduction of the amount of MDMX bound to p53.

To further elucidate the ability of RITA to affect p53/MDM2 and p53/MDMX 
complexes, we employed a yeast-based assay, which measures p53 transcriptional functionality using as a readout the activity of a p53-dependent luciferase reporter. Since p53 does not appear to be degraded by MDM2 in yeast cells, the inhibitory effect of MDM2 in this system can be ascribed to the direct interaction with p53 and the interference with p53dependent transactivation (Wang et al. 2001). Co-transfection of MDM2 inhibited the activity of p53 (Figure 3E), whereas RITA rescued wtp53-mediated transactivation of the reporter. Further, RITA protected p53 activity from inhibition by MDMX (Figure 3E).

Taken together, our results demonstrated that the allosteric effects exerted by RITA result in the inhibition of both p53/MDM2 and p53/MDMX interactions.

\section{Terminal hydroxyl groups of RITA are crucial for RITA/p53 interaction}

Our model implies that the central furan ring of RITA is not relevant for the binding with p53. Indeed, substitution of furan oxygen atom to sulphur (LCTA-2081, compound 2 see ST1 for structure) did not affect p53-dependent suppression of HCT 116 cancer cells' growth (Figure 4A). Further analysis of RITA analogs (ST1) let us to conclude that the presence of three rings is required for its $\mathrm{p} 53$-dependent biological activity.

Our molecular modeling predicts that the absence of one or two terminal hydroxyl groups should impair the interaction with p53. This prediction was supported by the loss of biological activity of RITA analog NSC-650973 (compound 4, ST1), lacking both hydroxyl groups (Figure 4A). Further, this compound did not compete with $\left[{ }^{14} \mathrm{C}\right]-\mathrm{RITA}$ for p53 binding in vitro (Figure 4B), indicating that it does not interact with p53 (Figure S2A).

Next, we compared the ability of RITA and compound 4 to prevent p53/MDM2 interaction in cells. p53/MDM2 complexes were visualized in breast cancer MCF7 and osteosarcoma U2OS cells by in situ proximity ligation assay (in situ PLA, (Söderberg et al., 2006) (Figure 4C). Presence of the fluorescence signals generated as a result of p53 and 
MDM2 being in proximity to each other was used as the readout for the formation of p53/MDM2 complexes. We observed a significant decline of the number of nuclear fluorescent dots in both cells lines upon RITA treatment, indicating the prevention of p53/MDM2 complexes. Unlike RITA, compound 4 did not decrease the number of fluorescent dots, suggesting that it does not inhibit p53/MDM2 interaction in cells (Figure 4D, upper panel). In line with these data, compound 4 did not induce p53 accumulation (Figure 4D, lower panel). Notably, another analog, compound 3, lacking only one hydroxyl group (ST1, Figure S2B) was more efficient in suppressing the growth of HCT 116 cells than compound 4 (NSC-650973), but still less potent than RITA (Figure 4A and Issaeva et al., 2004). Thus, we conclude that in agreement with the prediction from our model, both terminal hydroxyl groups of RITA and three thiofuran rings are required for the efficient binding to $\mathrm{p} 53$. The ability to bind $\mathrm{p} 53$ correlates with the prevention of $\mathrm{p} 53 / \mathrm{MDM} 2$ binding, induction of p53 and p53-dependent growth suppression.

Critical role of serine 33 and serine 37 for RITA/p53 interaction and induction of p53 level and transcriptional activity

To further validate our model, which predicted the crucial role of serines 33 and 37 for RITA interaction, we mutated serine 33 (S33) to alanine, alone or in combination with serine 37 (S37), and assessed the binding of RITA to Np53(S33A) and Np53(S33A/S37A) (referred to as p53 (33/37) peptide).

In line with our model, the interaction of both mutant proteins with RITA was decreased (Figure 5A), indicating the key role of these residues in binding to RITA.

Since mouse p53 lacks residues corresponding to serine 33 and proline 34 of human p53, we decided to test whether RITA can bind mouse p53 (Figure 5B). We detected only a weak interaction between RITA and mouse p53 N-terminal proteins (Figure 5C), 
suggesting that the presence of these residues is important for RITA binding. Further, in line with other approaches, Scintillation Proximity Assay (SPA), which detects the excitation of protein-coated beads by radioactively labeled RITA only when in a very close proximity (24), revealed that the binding of ${ }^{14} \mathrm{C}$-RITA to mouse p53 and $\mathrm{Np}(33 / 37)$ mutant is inefficient (Figure 5D).

In contrast to nutlin, which blocked p53/MDM2 complex and induced p53 accumulation in mouse cells, RITA did not disrupt the mouse p53/MDM2 interaction and did not induce p53 in mouse tumor cells and mouse embryonic fibroblasts (MEFs) expressing Ras and c-Myc oncogenes (Figure 5E and 5F). Nutlin but not RITA activated p53 beta-gal reporter in T22 mouse fibroblasts (Figure S3). These data are consistent with our previous results demonstrating the absence of growth suppression by RITA in mouse tumor cell lines (Issaeva et al., 2004).

Notably, swapping mouse p53 to human p53 in mouse embryo fibroblasts (SWAP MEF) derived from transgenic mice expressing human p53 in mouse p53-null background (Dudgeon et al., 2006) restored the ability of RITA to induce p53. As shown in Figure 5G, RITA induced p53 in SWAP MEF's expressing c-Myc and Ras. It did not affect SWAP cells without Ras and Myc overexpression, which is in line with our previous data suggesting that oncogene activation is required for RITA-mediated induction of p53 (Issaeva et al, 2004, Grinkevich et al, 2009). Taken together, these data suggest that S33 and, possibly P34 within SPLPS motif are required for RITA/p53 binding.

To further validate the role of Ser 33 and 37 we compared the ability of RITA to rescue wtp53 and S33A/S37A mutant (referred to as p53 (33/37) from MDM2 using yeastbased reporter assay. Both nutlin and RITA relieved p53-mediated transactivation from MDM2 inhibition (Figure 6A), whereas their effects on the activity of p53(33/37) were different. Nutlin protected both wt and p53(33/37) from inhibition by MDM2 equally well, 
but RITA had a significantly weaker effect on the p53(33/37) (Figure 6A). These data lend further support to the notion that S33 and S37 play an important role in RITA-mediated inhibition of p53/MDM2 interaction.

Next, we addressed the question whether the same serine residues are important for the induction of p53 in human cells by RITA. We stably expressed S33/S37 p53 and wtp53 in colon carcinoma RKO TP53-/- and SW48 TP53-/- cell lines, in which both alleles of wtp53 were inactivated by homologous recombination (Sur et al., 2009). Nutlin induced the accumulation of wt and p53(33/37) with similar efficiency (Figure 6B and not shown). In contrast, the induction of the double serine mutant by RITA was impaired (Figure 6B and not shown).

Importantly, CD spectroscopy confirmed our previously published data (Dickinson et al. 2015) that RITA promotes compaction of the random coil of Np53 (Figure 6C, left panel). These data support our idea that the binding of RITA to p53 triggers a conformational change in p53 (Figure 6C, left panel). To find out whether the alanine substitutions of serines 33 and 37 prevent the induction of conformational shift by RITA, we analyzed Np53(33/37) in CD spectroscopy. As shown in Figure 6C, (right panel), RITA did not promote more compact structure in Np53(33/37).

Moreover, ion mobility mass spectrometry (IMMS) provided a strong evidence for the induction of conformational change by RITA. IMMS is an attractive addition to native mass spectrometry as it allows study of the topology of proteins under various conditions (Jurneczko et al. 2013; Harvey et al. 2012). We performed IMMS analysis of both wt and Np53(33/37) in the presence and absence of RITA (Supplemental Experimental procedures). The wt Np53 in the presence of RITA presents as ions of the form $[\mathrm{M}+\mathrm{zH}]^{\mathrm{z+}}$ where $4 \leq z \leq 10$ with charge states $5 \leq z \leq 8$ at significant intensity (Figure 6D, upper panel). The mass spectra for Np53 without RITA, with RITA and the control spectra show no mass 
shift, suggesting that RITA binding is lost during desolvation (Figure S4A). Nonetheless, the conformation of Np53 in the presence of RITA was significantly altered. The collision cross section distributions in Figure 6D, show that in the absence of RITA, Np53 presents in two distinct conformational families centered at $\sim 1500$ and $\sim 1750 \AA^{2}$. Upon incubation with RITA the more extended conformer was lost, the conformer at $\sim 1500 \AA^{2}$ remained present at a lowered intensity and a third conformational family centered on $\sim 1000 \AA^{2}$ appeared, suggesting a significant compaction of the protein. Control experiments confirmed that this conformer was due to the presence of RITA (Figure S4A). This trend was observed for all sampled charge states (Figure S5A) with small variations in conformer intensity attributable to coulombic repulsion upon desolvation. Thus, small molecule RITA promotes generation of a unique compact conformer (or closely related conformational family) in wt Np53. This is in agreement with our previously published data showing that RITA promotes an allosteric change in p53 $\mathrm{N}$ terminus (Dickinson et al. 2015). In contrast, Np53(33/37) showed no conformational change upon RITA incubation (Figure 6D, bottom panel and Figure S4B, S5B). Np53(33/37) presents in two conformations centered at $\sim 1100$ and $1500 \AA^{2}$ both in the absence and presence of RITA. Taken together, these data provide compelling evidence that the allosteric activation of $\mathrm{p} 53$ by RITA requires residues S33 and S37.

Identification of PpIX as another allosteric activator of p53

Next we decided to assess whether the identified allosteric mechanism of p53 activation could be applied to other inhibitors of p53/MDM2 interaction. We have previously shown that small molecule protoporphyrin IX (PpIX), a photosensitizer applied in clinics, binds to the p53 N-terminus and disrupts p53/MDM2 complex (Zawacka-Pankau et al., 2007, Sznarkowska et al., 2010). Here, we tested whether PpIX targets the same amino acid residues in p53 as RITA, using fluorescent-based small-molecule band shift assay. Our data 
indicate that a mutation in serine 33 or double mutation in serine 33 and serine 37 decreases the binding of PpIX to the p53 N-terminus (Figure S6).

Next, we used a new technology called a Fluorescent-2 Hybrid (F2H®) Assay developed to study protein-protein interactions in live cells (Zolghard et al., 2008). Briefly, we employed tethering of MDM2 protein (LacI-GFP-Mdm2) at protein-protein interaction platform in the nucleus of U2OS cells and detected the complex of MDM2 with p53 (RFPp53) using fluorescent-based imaging (Figure S7A). We detected a potent inhibition of p53/MDM2 interaction in U2OS cells by PpIX (61 $\pm 8 \%, p<0.01, \mathrm{n}=6)$, which was comparable to positive control, nutlin $(60 \pm 5 \%, p<0.001, \mathrm{n}=6)$.

Using yeast-based reporter assay we confirmed that PpIX rescues p53 transcriptional activity from both MDM2 and MDMX (Figure S7B).

Taken together, our findings implicate the conformational state of the SPLPS sequence distal from the MDM2-interacting residues as a key structural element regulating p53/MDM2 interaction as presented in model in Figure 6E. This site could be modulated by small molecules such as RITA and PPIX.

\section{Identification of licofelone as an allosteric activator of p53}

Next we applied our findings for the identification of novel small molecules, which can prevent p53/MDM2 binding via the allosteric mechanism that we discovered. Therefore, we performed an informational search using the Therapeutic Targets Database (TTD) (URL:http://bidd.nus.edu.sg/group/cjttd/TTD_HOME.asp). We used the TTD internal Drug Similarity Search engine to estimate the Tanimoto coefficient (TC) of similarity for two molecules $A$ and $B$ (as described in Supplemental Experimental procedures). Our search resulted in the identification of several candidate compounds among which licofelone, a dual LOX/COX inhibitor, was found to be the top compound with TC of 0.73 (ST2). 
In order to select the candidate molecule for further studies, we performed function-related search of RITA and licofelone properties using tool which allows to predict the biological activity spectra based on the structural formula of the compounds (Lagunin et al. 2010; Filz et al. 2012). Our prediction analysis revealed that 17 out 26 (65.4\%) biological activities predicted for licofelone coincided with those predicted for RITA.

Thus, we selected licofelone for further validation in biological assays. We found that licofelone efficiently inhibited the growth of cancer cell lines (Figure 7A and B). Notably, this was p53-dependent, since licofelone did not suppress the growth of HCT 116 p53-/- cells (Figure 7B). Licofelone efficiently competed for the binding to p53 with radioactively-labeled RITA in gel shift assay, indicating that it binds the p53 N-terminus (Figure 7C). In line with p53-dependent growth suppression, licofelone induced p53 accumulation and expression of p53 pro-apoptotic targets Puma and Noxa in a p53-dependent manner (Figure 7D). Licofelone disrupted p53/MDM2 interaction as assessed by coimmunoprecipitation assay (Figure 7E), which supports its functional similarity to RITA.

To evaluate the role of the structural element which we found to be critical for the allosteric activation of p53, we tested whether alanine substitutions of S33 and S37 will prevent the effect of licofelone, using isogenic cells expressing either wtp53 or p53(33/37). Licofelone blocked the interaction between p53 and MDM2, but did not affect the p53(33/37)/MDM2 complex (Figure 7F), suggesting that residues 33 and 37 are important for licofelone-mediated effect. In line with these data, licofelone treatment induced wtp53, but not p53(33/37) accumulation in RKO cells (Figure 7G).

Finally, we tested if licofelone can prevent p53/MDMX interaction. As evident from Figure 7H, RITA, licofelone and PpIX, but not nutlin, blocked the p53/MDMX interaction in HCT116 cells. This indicates that targeting the identified structural element in p53 leads to its activation via a simultaneous inhibition of MDM2 and MDMX binding. 
In conclusion, the biological validation of licofelone, a known anti-inflammatory compound, confirmed our prediction that it acts on p53/MDM2 interaction via a newly discovered allosteric mechanism.

\section{Discussion}

Reconstitution of the p53 tumor suppressor leads to the preferential suppression of highly malignant lesions (Feldser et al., 2010 Junttilla et al., 2010). These data provide a strong support for the studies aimed at reactivation of p53 function. Several compounds targeting the p53/MDM2 interaction via steric hindrance are currently undergoing clinical trials (Hoe et al, 2014). High attrition rate of new candidate drugs in clinical trials demands the identification of novel compounds with distinct mode of action and elucidation of mechanisms regulating $\mathrm{p} 53 / \mathrm{MDM}$ binding for the efficient implementation of p53-targeting treatments into clinical practice.

Small molecule RITA activates wild-type p53. RITA inhibits p53/MDM2 interaction; however it is unique among known MDM2 inhibitors because it binds p53, but not MDM2 (Issaeva et al., 2004). Even though RITA has been reported to display p53-independent effects through the induction of the DNA damage (Wanzel et al., 2016), it is a valuable tool to explore the mechanism of wild-type p53 reactivation. Thus, here, we addressed the question how exactly the binding of RITA to p53 affects p53/MDM2 complex.

In this study we identified the site in the p53 N-terminus targeted by RITA and demonstrated that RITA promotes the generation of a compact conformer in Np53, using CD spectroscopy and IMMS. Modulation of protein conformation by a weak binding ligand has previously been shown by IMMS (Harvey et al., 2012). Interestingly, IMMS can detect the changes in p53 conformation induced by single point mutations in the p53 core domain 
(Jurneczko et al., 2013), analogous to the detection of conformational shift in Np53 induced by RITA by IMMS.

Molecular dynamic simulations suggest that residues 32-37, coinciding with RITAbinding site, might be involved in stabilization of a conformational state in which MDM2contacting residues F19, W23 and L26 of p53 are tacked inside the molecule (EspinozaFonesca, 2009). In line with this study, tryptophan fluorescence assay demonstrated that W23 is shielded from the solvent (Kar et al., 2002). Since X-ray structure of the MDM2 in complex with short p53 peptide (1YCQ.pdb) suggests that residues F19, W23 and L26 should be facing out in order to bind MDM2 as shown in Figure 3C (Kussie et al., 1996), it follows that the binding of 553 to MDM2 requires gross conformational changes. Importantly, recent study revealed that segments $23-31$ and 31-53 of the p53 N-terminus are involved in longrange interactions and can affect p53's structural flexibility upon MDM2 binding or upon phosphorylation of residues S33, S46 and T81. In particular, the authors identified nonrandom structural fluctuations at 31-53 segment, which are affected by MDM2 binding. Moreover, the phosphorylation of S33, S46 and T81 induces both local and remote structural changes, which are propagated to the MDM2 binding site in p53 (Lum et al., 2012; Huang et al., 2009). These data provide experimental evidence supporting our idea that restricting conformational mobility of segment involving residues 33-37 might serve to prevent p53/MDM2 interaction.

Figure 6E illustrates our model implying that p53 exists in a range of conformational states, which are present in cells in a dynamic equilibrium. Binding to MDM2 induces F19, W23 and L26 to be exposed and to fit into the p53-binding cleft of MDM2, causing the equilibrium to shift in favor of this conformation. Interaction of RITA with SPLPS stabilizes the alternative conformation, in which MDM2-contacting residues are trapped inside. In this way, the binding of RITA to p53 shifts the balance towards the p53 conformer with low 
affinity to MDM2.

Further, we found that PpIX, which we have shown to bind to p53 and to disrupt p53/MDM2 interaction, also requires serine 33 and 37 for its functioning as a p53 activator.

Allosteric mechanism of RITA and PpIX action is an unexpected turn in the development of inhibitors of p53/MDM2 interaction, which is mostly focused on steric hindrance mechanism (Hoe et al, 2014).

Intriguingly, RITA-contact residues S33/P34 are involved in the conformational change elicited by Pin1 prolyl-isomerase, an endogenous allosteric modulator for $\mathrm{p} 53$, leading to the dissociation of p53 from MDM2 (Mantovani et al., 2007). It is thus possible that the conformational change induced by RITA is similar to the one triggered by Pin1 in cells. Further support for this idea comes from the observations that both RITA and Pin1 cause the release of p53 from its inhibitor iASPP (Issaeva et al., 2004, Mantovani et al., 2007) and that Pin1 contributes to p53-mediated apoptosis induced by RITA (Sorrentino et al., 2013).

Here, we identified another p53 inhibitor, MDMX, whose interaction with p53 is hindered by allosteric mechanism. Our study unravels that RITA, PpIX and licofelon inhibit both MDM2 and MDMX binding to p53. This is in line with our data that, in contrast to nutlin, RITA is highly efficient in killing cancer cells with high expression of MDMX (Spinnler et al, 2011).

Our allosteric model of p53/MDM2 interaction allowed us to identify a potent functional analog of RITA, licofelone, which acts similar to RITA.

Anti-inflammatory compound licofelone, a potent 5-LOX/COX inhibitor has fewer side effects than conventional drugs (Kulkarni et al., 2008). Licofelone has been shown to suppress the growth of cancer cells in vitro and in vivo, which is attributed to 5-LOX/COX inhibition (Mohammed et al., 2011; Tavolari et al., 2008). Using chemoinformatics, we made a prediction of novel activity of licofelone, i.e., prevention of p53/MDM2 interaction. We 
confirmed this prediction, showing that licofelone can inhibit p53/MDM2 binding; residues S33/S37 are required for this effect of licofelone. Further, licofelone induced p53 and its target genes and suppressed the growth of cancer cells in a p53-dependent manner.

Prediction of new applications for existing drugs using computational methods has provided fascinating insights into the previously unknown pharmacology of these drugs (Keiser et al., 2009). Drug repositioning is an accelerated route of drug discovery, due to the established clinical and pharmacokinetic data. Identification of licofelone as a new inhibitor of p53/MDM2 and MDMX interaction might in a future initiate its application for cancer treatment.

Our data establish the allosteric mechanism of inhibition of p53/MDM2 and p53/MDMX interaction by small molecules as a viable strategy for drug discovery and identify the structural elements that may provide a basis for generation of new allosteric activators of $\mathrm{p} 53$ which might be valuable additions to the targeted therapeutic pharmacopoeia.

\section{Materials and methods}

\section{In situ proximity ligation assay (PLA)}

In situ PLA was performed according to the Duolink (Olink biosciences) protocol with modifications (see Supplemental Experimental procedures for details)

\section{Binding assays with $\left[{ }^{14}\right.$ C]-RITA}

For a small molecule-band shift assay purified proteins $(20 \mu \mathrm{M})$ or $80 \mu \mathrm{g}$ of total protein from cell lysates and $\left[{ }^{14} \mathrm{C}\right]$-RITA $(40 \mu \mathrm{M})$ were incubated in buffer B (50 mM Hepes, pH 7.0, $150 \mathrm{mM} \mathrm{NaCl}, 35 \%$ glycerol) at $37^{\circ} \mathrm{C} 30 \mathrm{~min}$ and separated in standard $\mathrm{TBE}$ or gradient native gels. Gels were stained to visualize proteins and radioactivity was measured using Phosphoimager Amersham Biosciences. 
SDS-PAGE separation of RITA/protein complexes was performed in $10 \%$ gel after brief heating of lysates in loading buffer. Proteins were depleted from cell lysates using anti-p53 DO-1 or anti-actin antibody (AC-15, Sigma), immobilized on protein A-conjugated DynaBeads (Invitrogene).

Co-immunoprecipitation of p53/MDM2 or p53/MDMX was performed as described (Issaeva et al, 2004). MDM2 in precipitates from mouse tumor cells MCIM SS cells expressing wtp53 (Magnusson et al., 1998) was detected by 4B2 antibody, a gift from Dr. S. Lain. MDMX antibody was from Bethyl laboratories.

\section{Scintillation Proximity Assay}

SPA PVT Protein A beads $(500 \mu 1 /$ sample) were incubated for $2 \mathrm{~h}$ with anti-GST antibodies (1:100). $0.1 \mu \mathrm{g} / \mu \mathrm{l}$ of studied protein in SPA buffer (GST, Np53, Np53(33/37)) was added to GST-coated SPA beads. $10 \mu \mathrm{l}\left[{ }^{14} \mathrm{C}\right]$-RITA $(52 \mu \mathrm{Ci})$ diluted 4 times in SPA buffer was added to protein samples $(1.3 \mu \mathrm{Ci})$. Unlabeled RITA was used as a cold substrate. SPA buffer was added to the final volume of $100 \mu \mathrm{l}$. Complexes were incubated for $1 \mathrm{~h}$ at $37^{\circ} \mathrm{C}$ and luminescence released by the $\left[{ }^{14} \mathrm{C}\right]$-RITA-excited beads was measured in standard microplate reader.

\section{Circular dichroism spectroscopy $(C D)$}

Proteins were incubated with RITA (reconstituted in 100\% isopropyl alcohol) at 1:2 molar ratio in 25 ammonium acetate at $37^{\circ} \mathrm{C}$ for $20 \mathrm{~min}$. Final concentration of IPA was $5 \%$. All CD spectra were acquired using JASCO instrument. $0.1 \mathrm{~cm}$ Hellma ${ }^{\circledR}$ cells were used in the far-UV region $260-195 \mathrm{~nm}$ at $21^{\circ} \mathrm{C}$. CD spectra were recorded with a $1 \mathrm{~nm}$ spectral bandwidth, $0.5 \mathrm{~nm}$ step size with scanning speed $200 \mathrm{~nm} / \mathrm{min}$. The spectra were recorded 5 times and the data are representative of at least 3 independent experiments.

Mass Spectrometry and Ion Mobility Mass Spectrometry (IMMS) 
Mass spectrometry and IMMS were made on an in-house modified quadrupole time-of-flight mass spectrometer (Waters, Manchester, UK) containing a copper coated drift cell of length $5 \mathrm{~cm}$. The instrument, its operation and its use in previous studies on p53 have been described elsewhere (McCullough et al., 2008; Jurneczko et al., 2013; Dickinson et al. 2015). Np53 was prepared at a concentration $50 \mu \mathrm{M}$ in $50 \mathrm{mM}$ Ammonium Acetate. Protein was incubated with RITA at a $1: 2$ molar ratio at $37^{\circ} \mathrm{C}$ for 30 minutes before analysis. $5 \%$ isopropyl alcohol was added to solubilize the ligand in aqueous solution, consistent with CD spectroscopy data. In all cases three repeats were taken, each on different days (For details see Supplemental Experimental procedures).

\section{Molecular Modeling}

Homology model of p53 was developed using the Rosetta server (Kim et al., 2004, 2005; Rohl et al., 2004; Chivian et al., 2006). Generated models were validated and fitted to the cryo-EM data (Okorokov et al., 2006). Domain fitting into the 3D map of p53 was performed automatically using UCSF Chimera package from the Resource for Biocomputing, Visualization, and Informatics at the University of California, San Francisco (supported by NIH P41 RR-01081), (http://www.cgl.ucsf.edu/chimera/) and further refined by UROX (http://mem.ibs.fr/UROX/). (For details see Supplemental Experimental procedures).

\section{Yeast-based reporter assay}

The yeast-based functional assay was conducted as previously described (Tomso et al. 2005). Briefly, the p53-dependent yeast reporter strain yLFM-PUMA containing the luciferase cDNA cloned at the ADE2 locus and expressed under the control of PUMA promoter (Inga et al. 2002) was transfected with pTSG-p53 (Resnick and Inga 2003), pRB-MDM2 (generously provided by Dr. R. Brachmann, Univ. of California, Irvine, CA, USA), or pTSG-p53 S33/37 mutant and selected on double drop-out media for TRP1 and HIS3. Luciferase activity was measured $16 \mathrm{hrs}$ after the shift to galactose-containing media (Inga et al. 2002) and the 
addition of $1 \mu \mathrm{M}$ RITA, PpIX or $10 \mu \mathrm{M}$ nutlin (Alexis Biochemicals), or DMSO. Presented are average relative light units and the standard errors obtained from three independent experiments each containing five biological repeats.

\section{Supplemental information}

Supplemental information includes Supplemental Experimental Procedures, Supplemental References, four supplemental figures and two tables.

\section{Conflict of interests}

The authors declare no conflict of interests

\section{Acknowledgements}

This work was supported by grants to G.S. from the Swedish Research Council, the Swedish Cancer Society and Ragner Söderberg Foundation. J. Z-P would like to acknowledge the grant from KI, SLL and StratCan, KI. We are greatly indebted to Protein Science Facility for protein purification, to all our colleagues who shared with us their reagents and cell lines. The authors are grateful to Yari Ciribilli, Bartosz Ferens, Anna Kostecka and Alicja Sznarkowska for technical assistance.

\section{References:}

Andreotti, V., Ciribilli, Y., Monti, P., Bisio, A., Lion, M., Jordan, J., Fronza, G., Menichini, P., Resnick, M.A., Inga, A. (2011). p53 transactivation and the impact of mutations, cofactors and small molecules using a simplified yeast-based screening system. PLoS One. 6(6): e20643 
Chivian, D., Baker, D. (2006). Homology modeling using parametric alignment ensemble generation with consensus and energy-based model selection. Nucleic Acids Res., 34: e112.

Dickinson, E. R., Jurneczko, E., Nicholson, J., Hupp, T. R., Zawacka-Pankau, J., Selivanova, G., Barran, P. E. (2015) The use of ion mobility mass spectrometry to probe modulation of the structure of p53 and of MDM2 by small molecule inhibitors. Frontiers in Molecular Biosciences, 2;39.

Dudgeon, C., Kek, C., Demidov, O.N., Saito, S., Fernandes, K., Diot, A., Bourdon, J.C., Lane, D.P., Appella, E., Fornace, A.J. Jr, Bulavin, D.V. (2006). Tumor susceptibility and apoptosis defect in a mouse strain expressing a human p53 transgene. Cancer Res. 15: 66(6) 2928-2936.

Enge, M., Bao, W., Hedström, E., Jackson, S., Moumen, A., and Selivanova, G. (2009). MDM2-dependent downregulation of $\mathrm{p} 21$ and hnRNP K provides a switch between apoptosis and growth arrest induced by pharmacologicaly activated p53. Cancer Cell 15(3), 171-183.

Espinoza-Fonesca, L.M. (2009). Leucine-rich hydrophobic clusters promote folding of the Nterminus of the intrinsically disordered transactivation domain of p53. FEBS Lett. 583(3), $556-560$.

Feldser, D.M., Kostova, K.K., Winslow, M.M., Taylor, S.E., Cashman, C., Whittaker, C.A., Sanchez-Rivera, F.J., Resnick, R., Bronson, R., Hemann, M.T., Jacks T. (2010) Stagespecific sensitivity to p53 restoration during lung cancer progression. Nature. 468(7323), $572-5$. 
Filz, O.A., Lagunin, A.A., Filimonov, D.A. and Poroikov, V.V. (2012) In Silico fragmentbased drug design using a PASS approach. SAR and QSAR in Environmental Research. Volume 23, Issue 3-4, P. 279-296.

Grinkevich, V., Issaeva, N., Hossain, S., Pramanik, A., Selivanova, G. (2005). Reply to 'NMR indicates that the small molecule RITA does not block p53-MDM2 binding in vitro'. Nat Med. 11, 1136-1137.

Grinkevich, V., Nikulenkov, F., Shi, Y., Enge, M., Bao, W., Maljukova, A., Sangfelt, O., Gluch, A,, Kel, A., and Selivanova G. (2009). Ablation of key oncogenic pathways by RITAreactivated p53 is required for efficient apoptosis. Cancer Cell. 15(5), 441-453

Harvey, S. R., Porrini, M., Stachl, C., MacMillan, D., Zinzalla, G., Barran, P.E. (2012) Small-Molecule Inhibition of c-MYC:MAX Leucine Zipper formation Is Revealed by Ion Mobility Mass Spectrometry. J. Am. Chem. Soc. 134, 19384-19392

Hoe K.K., Verma C.S, Lane D.P. (2014). Drugging the p53 pathway: understanding the route to clinical efficacy. Nat Rev Drug Discovery, 13:217-236

Huang, F., Rajagopalan, S., Settanni, G., Marsh, R.J., Armoogum, D.A., Nicolaou, N., Bain, A.J., Lerner, E., Haas, E., Ying, L., Fersht, A.R. (2009). Multiple conformations of fulllength p53 detected with single-molecule fluorescence resonance energy transfer. Proc Natl Acad Sci U S A. 106:49, 20758-20763. 
Issaeva, N., Bozko, P., Enge, M., Protopopova, M., Verhoef, L.G., Masucci, M., Pramanik, A., and Selivanova, G. (2004). Small molecule RITA binds to p53, blocks p53-HDM-2 interaction and activates p53 function in tumors. Nat. Med. 10, 1321-1328.

Junttila, M.R., Karnezis, A.N,, Garcia, D., Madriles, F., Kortlever, R.M., Rostker, F., Brown Swigart, L., Pham, D.M., Seo, Y., Evan, G.I., Martins, C.P. (2010) Selective activation of p53-mediated tumour suppression in high-grade tumours. Nature. 468(7323), 567-71.

Jurneczko, E., Cruickshank, F., Porrini, M., Clarke, D. J., Campuzano, I. D. G., Nikolova, P. V., Barran, P. E. (2013) Probing the Conformational Diversity of Cancer-Associated Mutations in p53 with Ion-Mobility Mass Spectrometry. Angew. Chem. Int. Ed. 52, 43704374

Kar, S., Sakaguchi, K., Shimohigashi, Y., Samaddar, S., Banerjee, R., Basu, G., Swaminathan, V., Kundu, T.K., Roy, S. (2002). Effect of Phosphorylation on the Structure and Fold of Transactivation Domain of p53. J. Biol. Chem. 277, 15579 - 15585.

Keiser, M.J., Setola, V., Irwin, J.J., Laggner, C., Abbas, A.I., Hufeisen, S.J., Jensen, N.H., Kuijer, M.B., Matos, R.C., Tran, T.B., Whaley, R., Glennon, R.A., Hert, J., et al. (2009) Predicting new molecular targets for known drugs. Nature. 462(7270):175-81.

Kim, D.E., Chivian, D., Baker, D. (2004). Protein structure prediction and analysis using the Robetta server. Nucleic Acids Res. 32, W526 - W531. 
Kim, D.E., Chivian, D., Malmström, L., David Baker, D. (2005). Automated prediction of domain boundaries in CASP6 targets using Ginzu and RosettaDOM. Proteins: Structure, Function, and Bioinformatics. 61, 193-200.

Koehler, M.F., Zobel, K., Beresini, M.H., Caris, L.D., Combs, D., Paasch, B.D., Lazarus, R.A. (2002). Albumin affinity tags increase peptide half-life in vivo. Bioorg Med Chem Lett. 12(20), 2883-2886.

Krajewski, M., Ozdowy, P., D'Silva, L., Rothweiler, U., Holak, T.A., (2005). NMR indicates that the small molecule RITA does not block p53-MDM2 binding in vitro. Nat. Med. 11(11), $1135-1136$.

Kulkarni, S.K., Singh, V.P. (2008) Licofelone: the answer to unmet needs in osteoarthritis therapy? Curr Rheumatol Rep. 10(1):43-8.

Kussie, P.H., Gorina, S., Marechal, V., Elenbaas, B., Moreau, J., Levine, A.J., Pavletich, N.P. (1996). Structure of the MDM2 oncoprotein bound to the p53 tumor suppressor transactivation domain. Science. 274(5289), 948-953.

Lagunin, A., Filimonov, D.A., Poroikov, V.V. (2010) Multi-targeted natural products evaluation based on biological activity prediction with PASS. Cur. Phar. Des. 16 (15), $1703-$ 1717. 
Lowry, D.F., Hausrath, A.C., Daughdrill, G.W. (2008). A robust approach for analyzing a heterogenous structural ensemble. Proteins: Structure, Function, and Bioinformatics. 73, 918-928.

Mohammed, A., Janakiram, N.B., Li, Q., Choi, C.I., Zhang, Y., Steele, V.E., Rao, C.V. (2011) Chemoprevention of colon and small intestinal tumorigenesis in APC(Min/+) mice by licofelone, a novel dual 5-LOX/COX inhibitor: potential implications for human colon cancer prevention. Cancer Prev Res (Phila). 4(12):2015-26.

MacroModel, version 9.6; Schrödinger, LLC:New York, NY, 2008.

Magnusson, K.P., Satalino, R., Qian, W., Klein, G., Wiman, K.G. (1998). Is conversion of solid into more anoxic ascites tumors associated with p53 inactivation? Oncogene 17, 2333 2337.

Mantovani, F., Tocco, F., Girardini, J., Smith, P., Gasco, M., Lu, X., Crook, T., Del Sal, G., (2007). The prolyl isomerase Pin1 orchestrates p53 acetylation and dissociation from the apoptosis inhibitor iASPP. Nat Struct Mol Biol. 14, 9212-9220.

McCullough, B. J., Kalapothakis, J., Eastwood, H., Kemper, P., MacMillan, D., Taylor, K., Dorin, J., Barran, P.E. (2008) Development of an Ion Mobility Quadropole Time of Flight Mass Spectrometer. Anal. Chem. 80, 6336-6344 
Okorokov, A.L. Sherman, M.B., Plisson, C., Grinkevich, V., Sigmundsson, K., Selivanova, G., Milner, J., Orlova, E.V. (2006). The structure of p53 tumour suppressor protein reveals the basis for its functional plasticity. EMBO J. 25(21), 5191-5200.

Rohl, C.A., Strauss, C.E.M., Chivian, D., Baker, D. (2004). Modeling structurally variable regions in homologous proteins with rosetta. Proteins: Structure, Function, and Bioinformatics. 55, 656-677.

Sorrentino, G., Mioni, M., Giorgi, C., Ruggeri, N., Pinton, P., Moll, U., Mantovani F, Del Sal G. (2013). The prolyl-isomerase Pin1 activates the mitochondrial death program of p53. Cell Death Differ. 20(2), 198-208.

Söderberg, O., Gullberg, M., Jarvius, M., Ridderstråle, K., Leuchowius, K.J., Jarvius, J., Wester, K., Hydbring, P., Bahram, F., Larsson, LG., Landegren, U. (2006). Direct observation of individual endogenous protein complexes in situ by proximity ligation. Nat. Methods. 3, 995-1000.

Sur, S., Pagliarini, R., Bunz, F., Rago, C., Diaz, L.A. Jr., Kinzler, K.W., Vogelstein, B., Papadopoulos, N. (2009). A panel of isogenic human cancer cells suggests a therapeutic approach for cancers with inactivated p53. Proc Natl Acad Sci U S A. 106(10), 3964-3996.

Shangary, S., Ding, K., Qiu, S., Nikolovska-Coleska, Z., Bauer, J.A., Liu, M., Wang, G., Lu, Y., McEachern, D., Bernard, D., Bradford, C.R., Carey, T.E., Wang, S. (2008). Reactivation of p53 by a specific MDM2 antagonist (MI-43) leads to p21-mediated cell cycle arrest and selective cell death in colon cancer. Mol Cancer Ther. 7, 1533-1542. 
Sznarkowska, A., Maleńczyk, K., Kadziński, L., Bielawski, K.P., Banecki, B., ZawackaPankau, J. (2011) Targeting of p53 and its homolog p73 by protoporphyrin IX. FEBS Lett. 585(1):255-260.

Tavolari, S., Bonafè, M., Marini, M., Ferreri, C., Bartolini, G., Brighenti, E., Manara, S., Tomasi, V., Laufer, S., Guarnieri, T. (2008) Licofelone, a dual COX/5-LOX inhibitor, induces apoptosis in HCA-7 colon cancer cells through the mitochondrial pathway independently from its ability to affect the arachidonic acid cascade. Carcinogenesis 29(2), $371-80$.

Toledo, F., Wahl, G.M. (2007) MDM2 and MDM4: p53 regulators as targets in anticancer therapy. Int J Biochem Cell Biol. 39, 1476-1482.

Vousden, K.H., Prives, C. (2009) Blinded by the Light: The Growing Complexity of p53. Cell. 137(3):413-31.

Yang, Y., Ludwig, R.L., Jensen, J.P., Pierre, S.A., Medaglia, M.V., Davydov, I.V., Safiran, Y.J., Oberoi, P., Kenten, J.H., Phillips, A.C. et al. (2005). Small molecule inhibitors of HDM2 ubiquitin ligase activity stabilize and activate p53 in cells. Cancer Cell. 7(6), 547559.

Zawacka-Pankau, J., Issaeva, N., Hossain, S., Pramanik, A., Selivanova, G., Podhajska, A.J. (2007) Protoporphyrin IX interacts with wild-type p53 protein in vitro and induces cell death 
of human colon cancer cells in a p53-dependent and -independent manner. J Biol Chem.

282(4), 2466-2472.

Wanzel, M., Vischedyk, J.B., Gittler, M.P., Gremke, N., Seiz, J.R., Hefter, M., Noack,

M., Savai, R., Mernberger, M., Charles, J.P., Schneikert, J., Bretz, A.C., Nist, A., Stiewe, T.

(2016) CRISPR-Cas9-based target validation for p53-reactivating model compounds. Nat

Chem Biol. 12(1):22-8.

\section{Figure legends}

Figure 1. RITA binds $\mathbf{p 5 3}$ in cells and in vitro.

A. ${ }^{14}$ C-RITA/protein complexes were detected in lysates of HCT 116 or HCT 116 TP53/cells treated with $5 \mu \mathrm{M}\left[{ }^{14} \mathrm{C}\right]$-RITA for $12 \mathrm{~h}$ upon separation in $10 \%$ SDS-PAGE under mild denaturing conditions. The position of RITA in the gel was visualized by autoradiography. p53 was detected by immunoblotting with DO-1 antibody (right panel). Shown is a representative data of three independent experiments.

B. A small-molecule band shift assay was used to detect $\left[{ }^{14} \mathrm{C}\right]-\mathrm{RITA} /$ protein complexes in lysates of HCT116 or HCT116TP53-/- cells treated with $\left[{ }^{14} \mathrm{C}\right]-\mathrm{RITA}$, and separated in gradient polyacrylamide gel under native conditions. $\left[{ }^{14} \mathrm{C}\right]$-RITA and p53 were detected as in $\mathbf{A}$.

C. $\left[{ }^{14} \mathrm{C}\right]-$ RITA interaction with purified proteins, GST-Np53(2-65), fibrinogen and human serum albumin (HSA) was detected by band shift assay using 2:1 molar excess of RITA. Dotted line indicates where the gel was cut.

D. Upon standard SDS electrophoresis $\left[{ }^{14} \mathrm{C}\right]-\mathrm{RITA} /$ protein complexes were disrupted $(\mathrm{n}=3)$.

Figure 2. Mapping of RITA binding site within the p53 N-terminus. 
A. Scheme depicting the series of deletion mutants of GST-Np53 used in this study.

B. The binding of $\left[{ }^{14} \mathrm{C}\right]$-RITA to $\mathrm{p} 53 \mathrm{~N}$ terminus deletion mutants were analyzed in band shift assay as in Figure 1C.

Figure 3. Molecular model of the p53/RITA complex and inhibition of p53/MDMX interaction by RITA.

A. Binding of RITA to SPLPS sequence (cyan) of p53 involves interaction with S33 and S37 via terminal hydroxyl groups of RITA, and hydrophobic interactions with P34 and P36. Hydrogen bonds are highlighted in black dotted lines. Orientation of MDM2-binding helix of p53 (lime) is different upon p53 binding to RITA (blue) B. and to MDM2 (purple) (pdb: 1YCQ) (c). Side chains of residues (F19, W23 and L26) involved in MDM2 binding are shown in (B., C.). Atom type coloring; oxygen (red), nitrogens (blue), and sulphurs (yellow). See also Movie S1.

D. In line with the model prediction, RITA-mediated p53 conformational change results in the inhibition of p53/MDMX binding in HCT 116 cells as assessed by coimmunoprecipitation. This is representative data of three independent experiments.

E. RITA rescues the p53 transcriptional activity from inhibition by MDM2 or MDMX as assessed in yeast-based functional assay. The average light units relative to the transactivation activity of p53 alone and the standard errors of at least five biological repeats are presented. The $t$-student test was performed for statistical analysis with $\mathrm{p} \leq$ 0.05 .

Figure 4. Two terminal hydroxyl groups of RITA are crucial for p53 binding and disruption of p53/MDM2 interaction. 
A. RITA analog NSC-650973 (compound 4) lacking two hydroxyl groups did not suppress the growth of HCT 116 cancer cells, unlike LCTA-2081 (compound 2) analog with substituted $\mathrm{O}$ atom in furan ring (for structure refer to Supplementary Table 1), which retained full biological activity. NSC-672170 (compound 3) analog with one hydroxyl group substituted to ketone retained more then $60 \%$ of RITA biological activity.

B. Biologically inactive RITA analog compound 4 did not compete for the binding to GSTNp53 with $\left[{ }^{14} \mathrm{C}\right]$-RITA.

C. Visualization of $\mathrm{p} 53 / \mathrm{MDM} 2$ complexes in MCF7 and U2OS cells treated or non-treated with RITA using in situ Proximity Ligation Assay (in situ PLA) allowed to detect a decreased number of p53/MDM2 complexes per cell (red fluorescent dots) upon RITA treatment.

D. Quantification of in situ PLA results demonstrated the decline of average number of nuclear signals upon treatment with RITA, but not its derivative NSC-650973 (upper panel). The normality was assessed with Shapiro-Wilk's test. $\mathrm{p}<0.05$ values were considered statistically significant. RITA, but not compound 4 induced p53 accumulation in HCT116 cells, as detected by immunoblotting (lower panel).

Figure 5. Serine 33 and serine 37 are crucial for the efficient binding of RITA to the p53 $\mathbf{N}$ terminus.

A. Assessment of $\left[{ }^{14} \mathrm{C}\right]$-RITA interaction with $\mathrm{Np} 53$ proteins carrying alanine substitutions of S33 or S33/S37 using band shift assay as in Figure 1C.

B. Alignment of mouse and human p53 N-termini, highlighting the site for MDM2 interaction and RITA-binding motif. 
C. D. Inefficient binding of RITA to GST-fusion mouse p53 proteins, spanning residues 164 and 1-85 as detected by band shift assay and scintillation proximity assay (SPA). SPA assay reveals inefficient binding of RITA to Np53(33/37).

E. Co-immunoprecipitation experiment demonstrated that RITA did not prevent MDM2/p53 interaction in TA3-Sth mouse cancer cells, unlike nutlin. C, control untreated sample, R, RITA-treated and N, nutlin-treated samples, dotted line represents different exposure time of this part of the membrane.

F. Mouse p53 in Myc- and Ras-transformed MEF's was not induced by RITA in contrast to nutlin.

G. RITA efficiently induced the level of human p53 in SWAP MEF's transfected with Ras and c-Myc as detected by immunoblot.

Figure 6. Serine 33 and serine 37 are required for RITA-mediated rescue of p53 from MDM2-dependent inhibition.

A. The effect of the co-expression of MDM2 along with wt or 33/37 p53 upon the treatment with $1 \mu \mathrm{M}$ RITA $(\mathrm{R})$ or $10 \mu \mathrm{M}$ nutlin $(\mathrm{N})$ as assessed in yeast-based luciferase reporter assay. The average light units relative to the transactivation activity of p53 alone and the standard errors of at least five biological repeats are presented.

B. Induction of the p53 protein levels by RITA and nultin3a in colon cancer cells RKO and their p53-null derivatives with reconstituted wt p53 and p53(33/37) as assessed by Western blotting.

C. RITA promotes more compact structure of random coil in wt Np53 (left) but not in Np53(33/37) (right) as detected by circular dichroism spectroscopy (CD).

D. nESI mass spectra (left) and drift tube ion mobility mass spectrometry collision cross section distributions arising from arrival time distributions (right) for the $[\mathrm{M}+6 \mathrm{H}]^{6+}$ 
analyte of wt Np53 in the absence and presence of RITA (top panel, adapted from (Dickinson et al. 2015)) and Np53(33/37) in the absence and presence of RITA (bottom panel). Conformational families are depicted by coloured Gauusian curves. wt Np53 undergoes a compaction event resulting in the induction of a novel conformational family shown in red. Np53(33/37) conformational spread is unaffected by RITA induction.

E. A scheme illustrating allosteric mechanism of RITA-mediated prevention of p53/MDM2 interaction. Binding of RITA shifts the balance towards p53 conformation with low affinity to MDM2.

Figure 7. Licofelone activates p53 and disrupts p53/MDM2 and p53/MDMX interactions via targeting serine 33 and 37 residues.

A. Dose-dependent inhibition of proliferation by licofelone in MCF7 cells as examined in WST assay.

B. Licofelone suppressed the growth of human colon cancer cells in a p53-dependent manner.

C. Licofelone competed for the binding to Np53 with ${ }^{14} \mathrm{C}$-RITA in small molecule bandshift assay

D. Licofelone induced p53 and its targets PUMA and Noxa in HCT 116 and MCF7.

E. p53/MDM2 complex disruption in HCT 116 cells by licofelone as assessed in coimmunoprecipitation assay.

F. Induction of the p53 protein by licofelone in p53-null RKO colon cancer cells with reintroduced wt p53, but not in cells expressing p53(33/37) as assessed by Western blotting. 
G. Licofelone disrupted the interaction between MDM2 and wtp53 but not p53(33/37) mutant p53 in RKO cells as examined by co-immunoprecipitation

H. RITA, PpIX and licofelone, but not nutlin, disrupted p53/MDMX interaction in HCT 116 cells assessed as in $\mathbf{G}$. 
A.

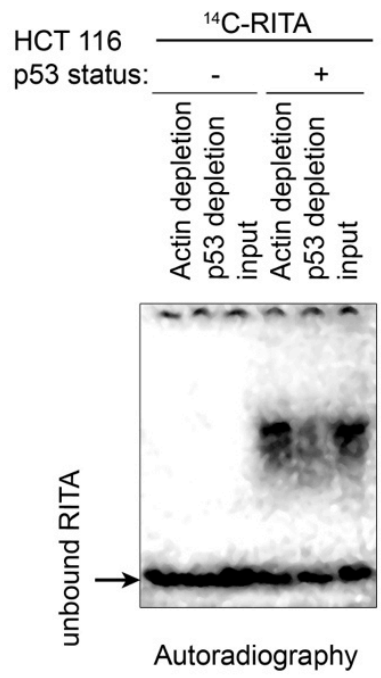

C.

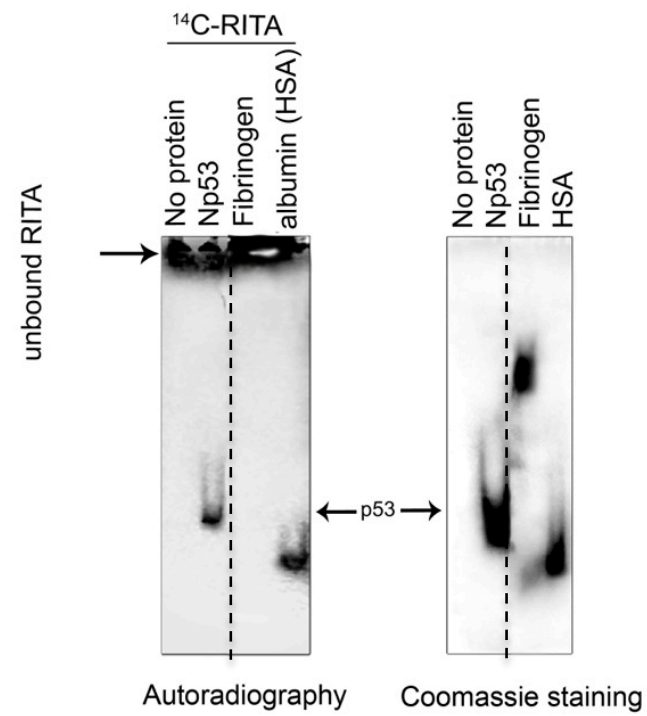

B.

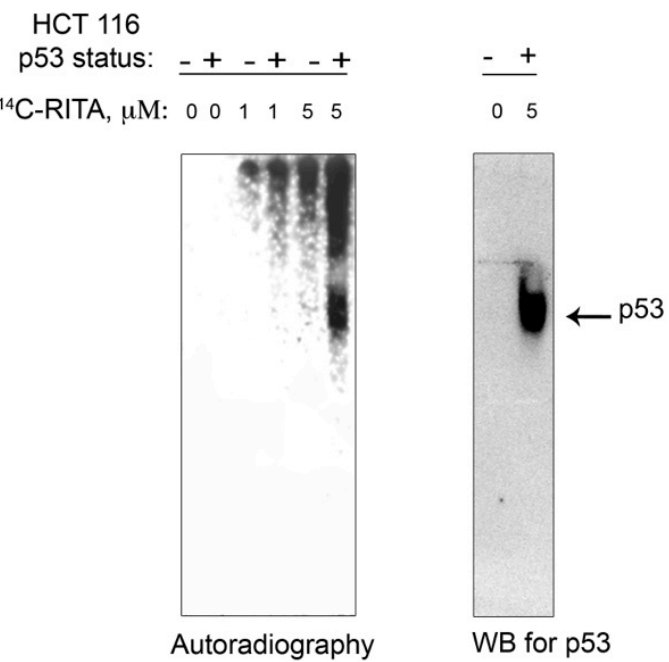

D.

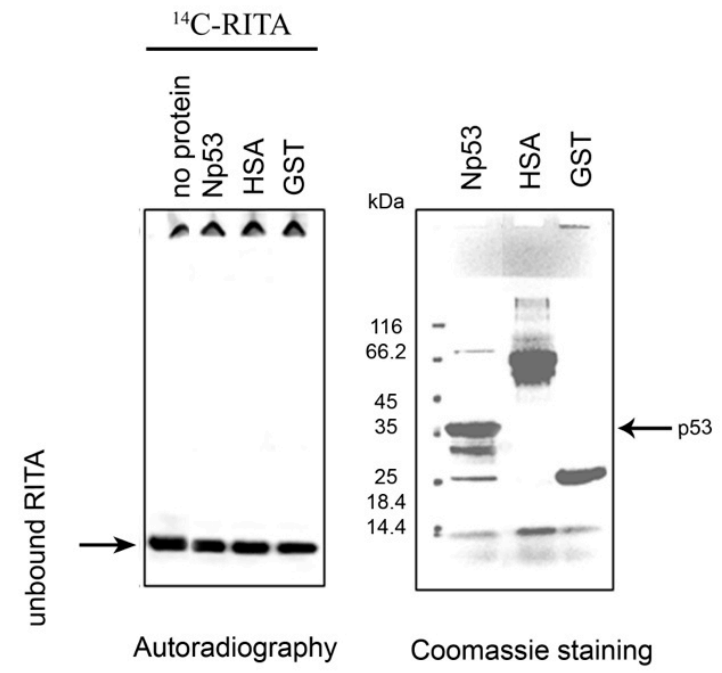


A.

p53 constructs

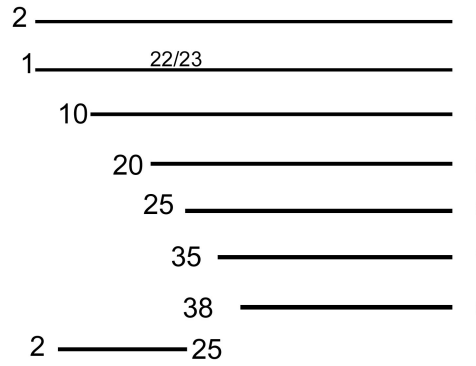

RITA binding

$65 \quad++$

$65++$

$65++$

$65++$

$65++$

$65+$
58 -

B.

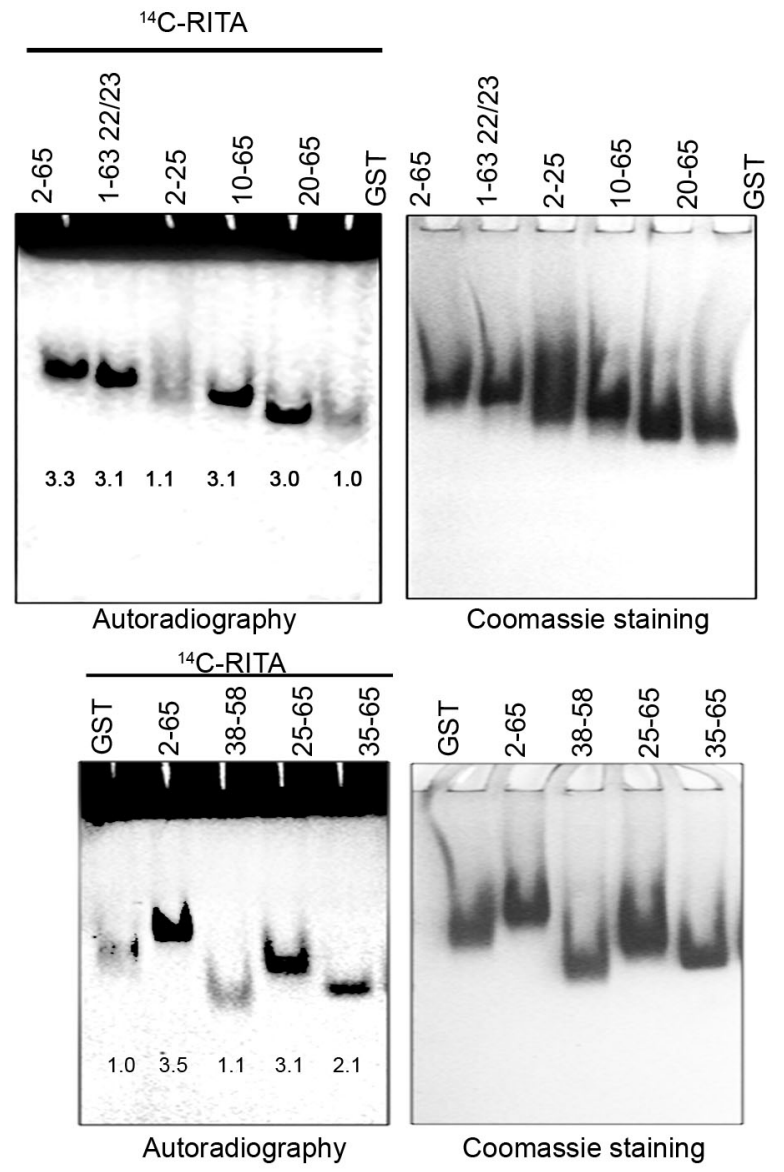


A.

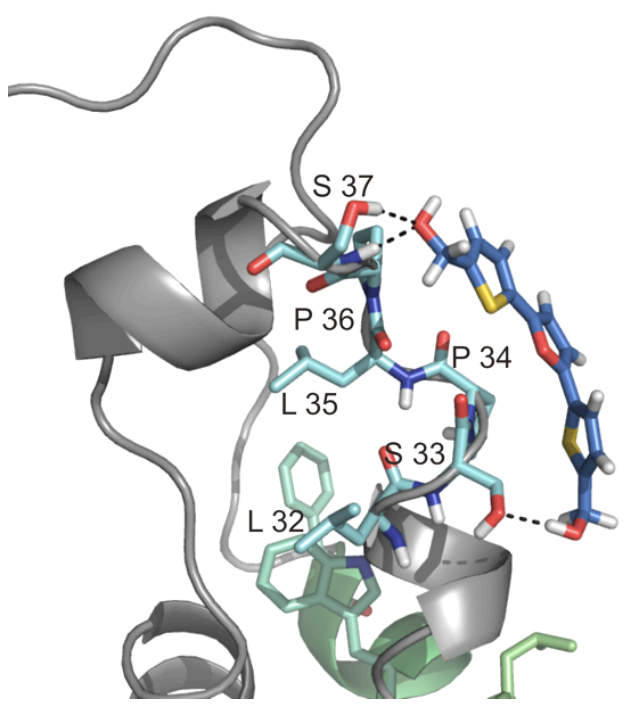

C.

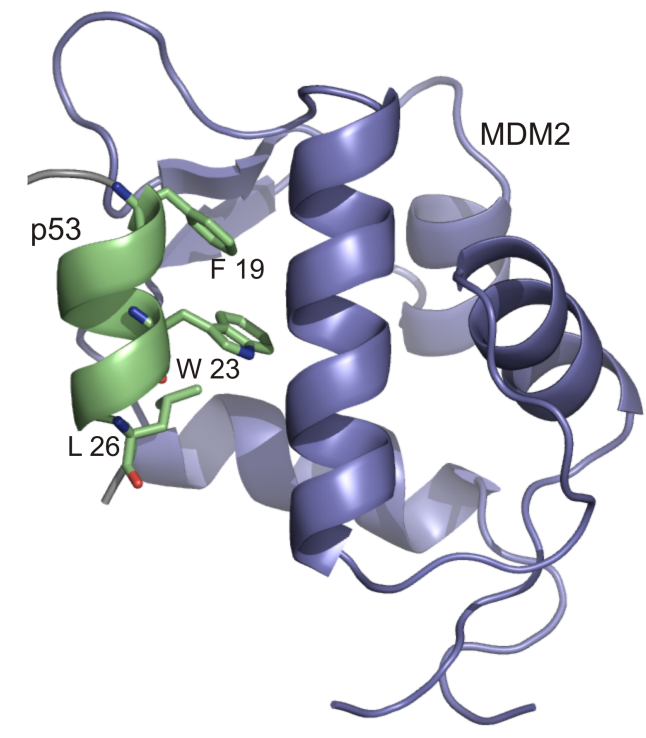

E.

Yeast-based reporter assay

$\square$ p53

$\square$ p53+MDM2

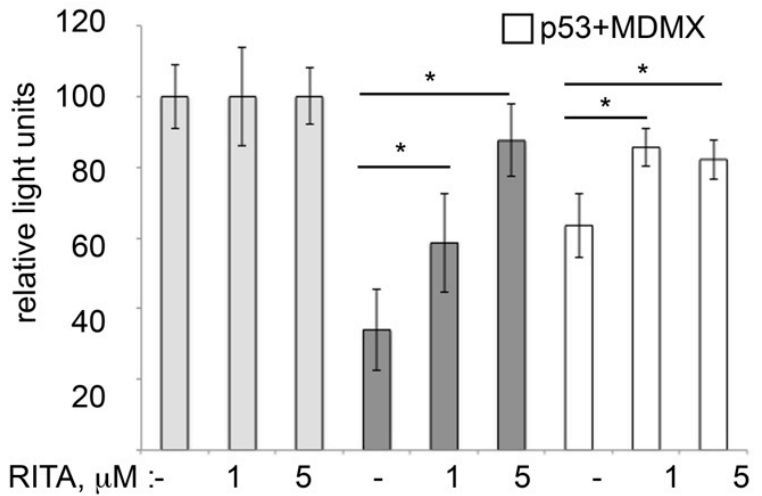

B.

D.

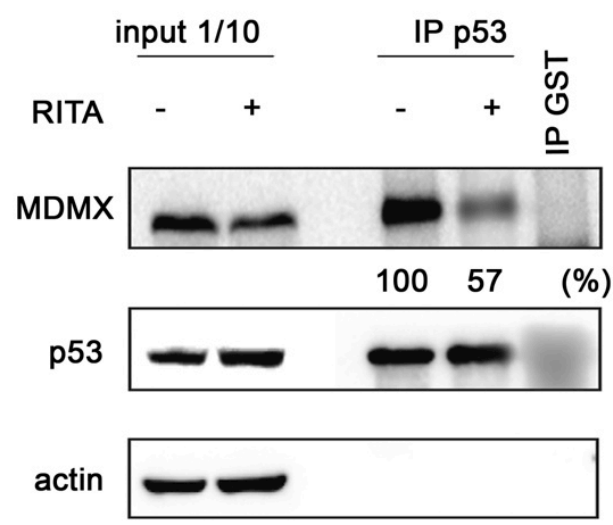


A.
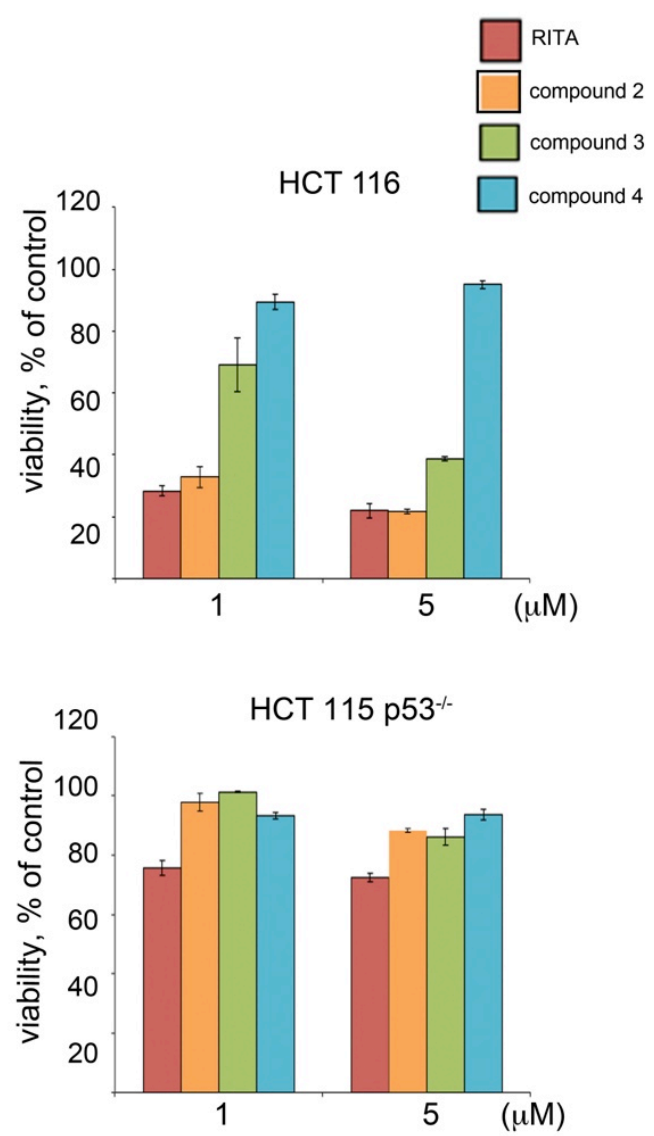

c.

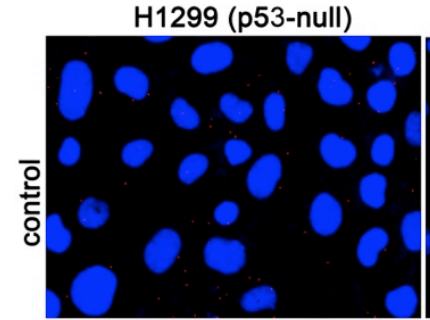

U2OS w/o secondary Ab
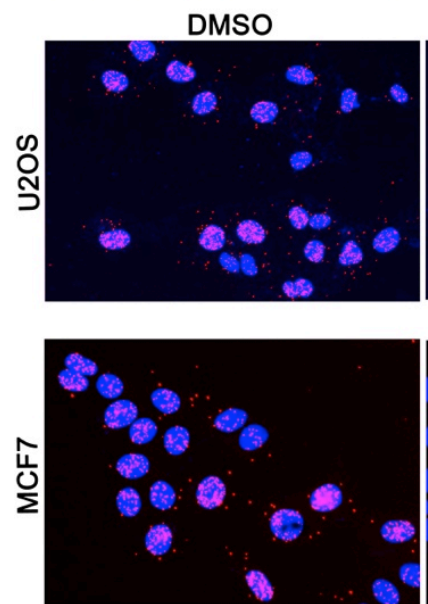

B.

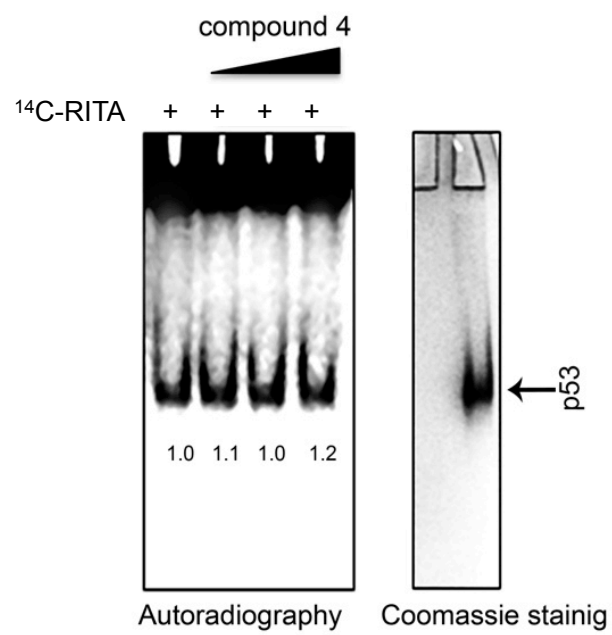

D.
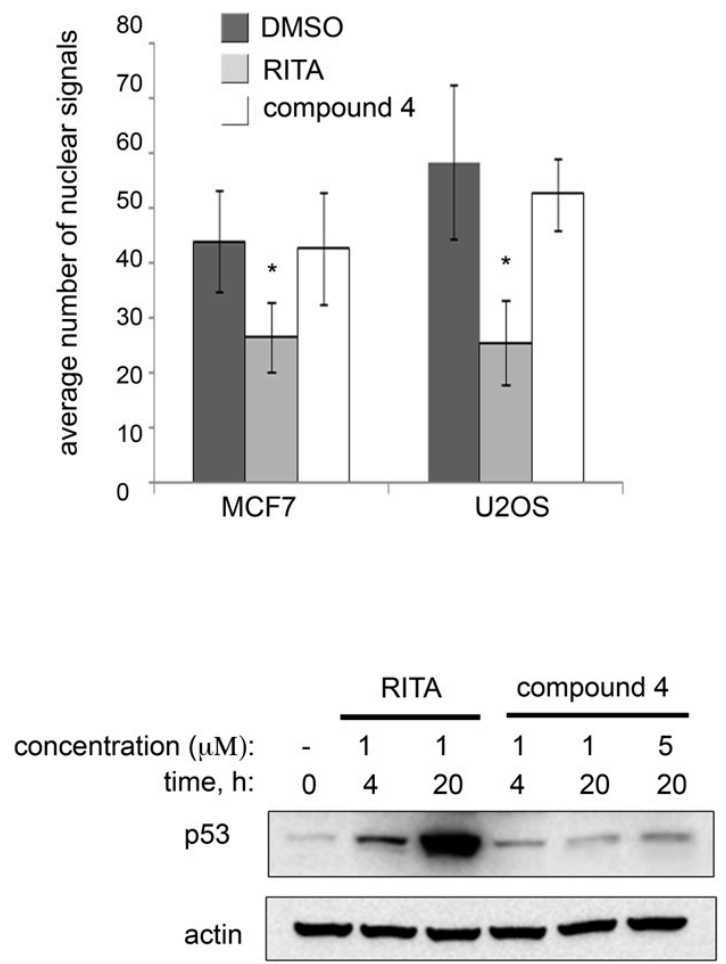
A.

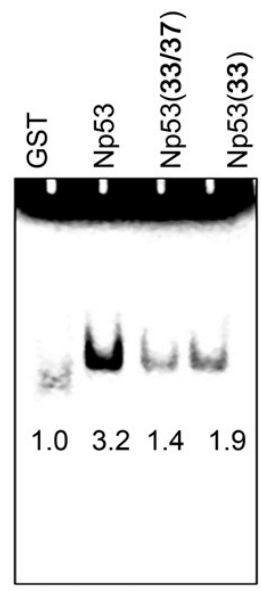

Autoradiography

C.

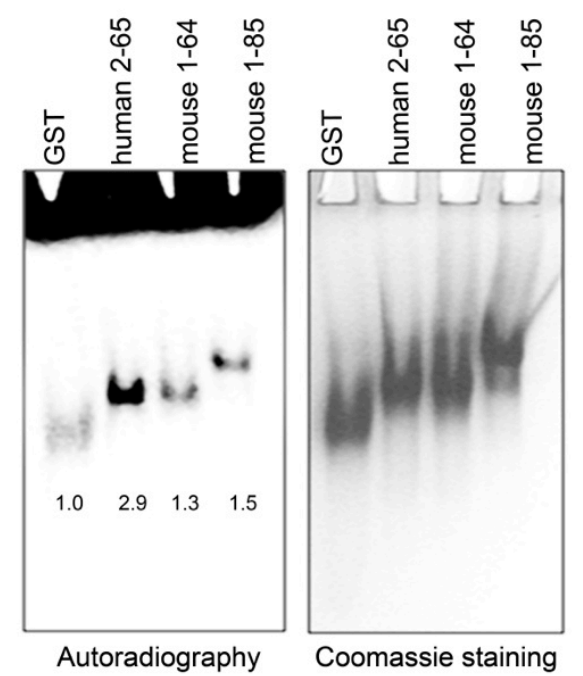

E.

TA3-Sth mouse cancer cells

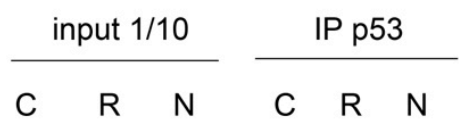
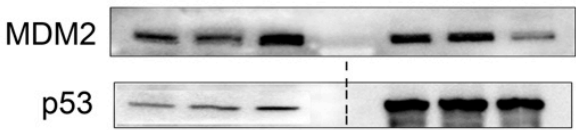

G.

SWAP MEF SWAP MEF Myc+Ras

\begin{tabular}{|c|c|c|c|c|c|c|}
\hline $\operatorname{RITA}(\mu \mathrm{M})$ & - & 1 & 5 & - & 1 & 5 \\
\hline p53 & - & - & - & - & - & - \\
\hline
\end{tabular}

B.

\section{p53 H. sapiens 10 EPPLSQETF SDLWKLLPENNVLSPLPSQAM 13020 p53 M. musculus ELPLSQETF SGLWKLLPPEDILPSPHCMDD}

D.

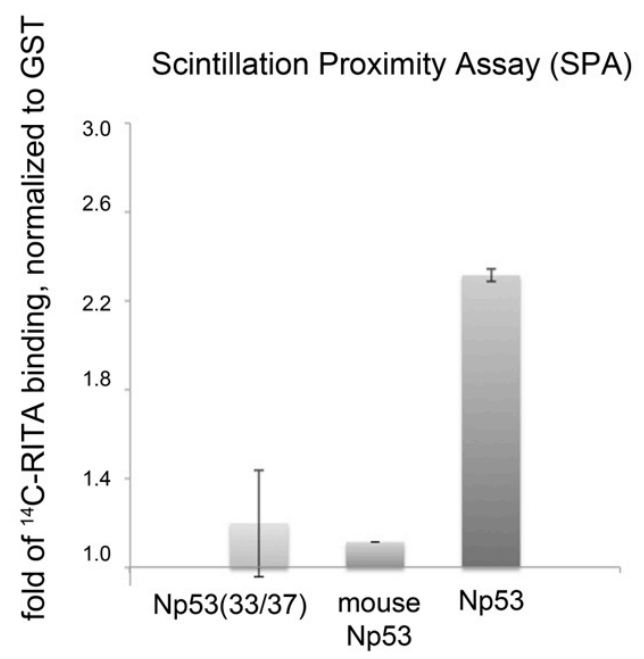

F. MEF Myc + Ras

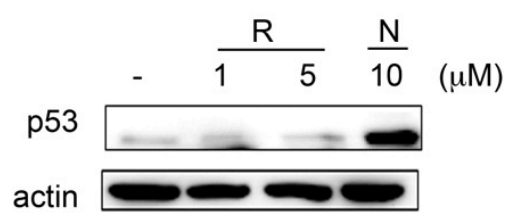


A.

c.
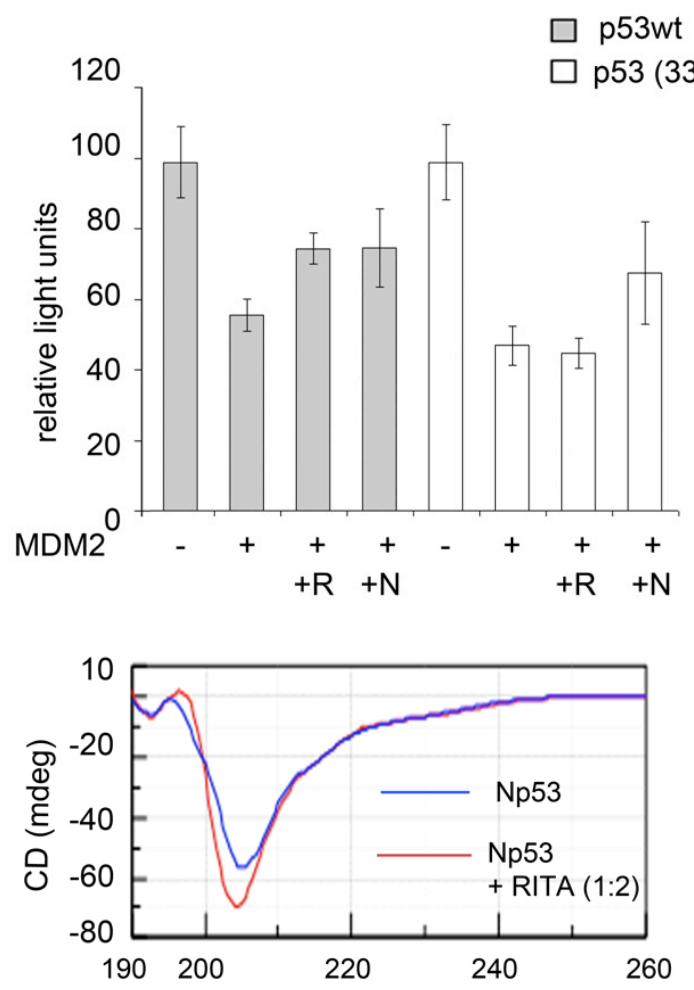

(nm)

D.
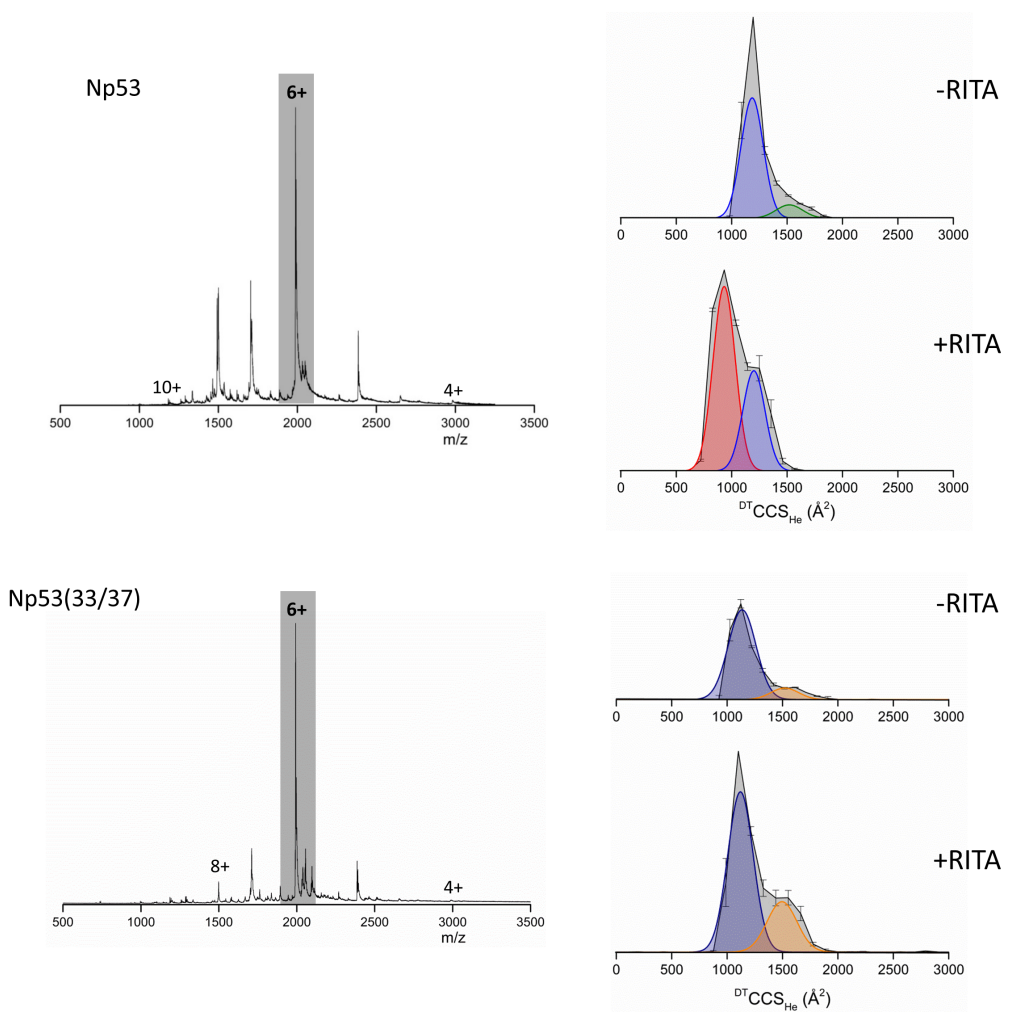

RKO
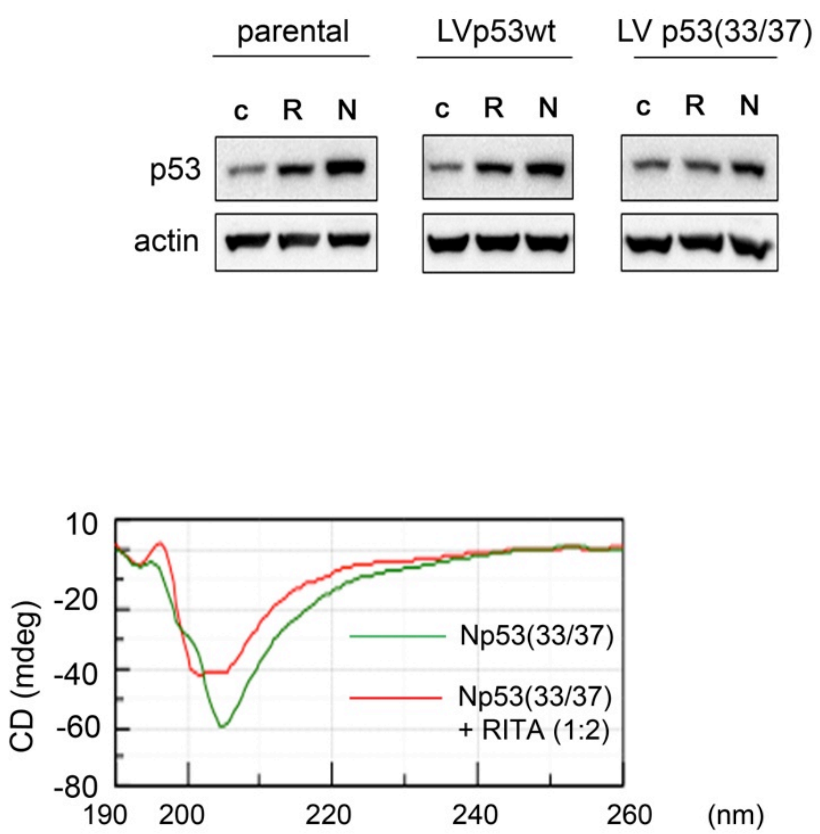

E.<smiles>CC[As]1CCC1(CC)CC</smiles><smiles>OOOOO[Mg]</smiles><smiles>CCC1(CC)CC[As]1C1CC1</smiles><smiles>c1ccc2ccccc2c1</smiles><smiles>CCC1(CC)CCCCC1</smiles><smiles>CCC12CCCC(CC)(CC1)OO2</smiles> 
A.

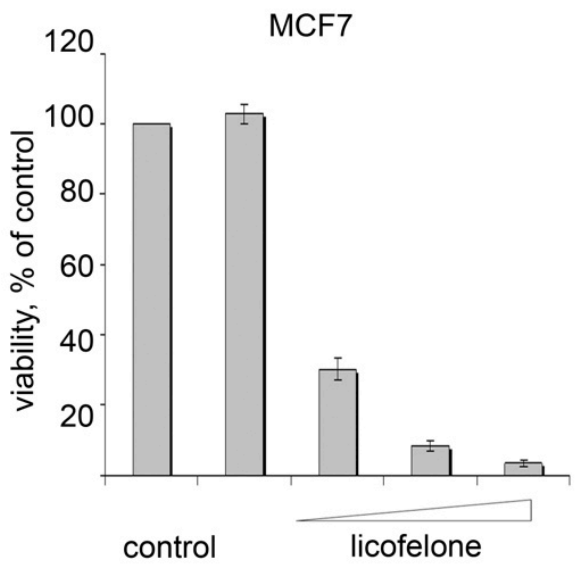

D.

MCF7
HCT $116 \quad$ HCT 116 p53

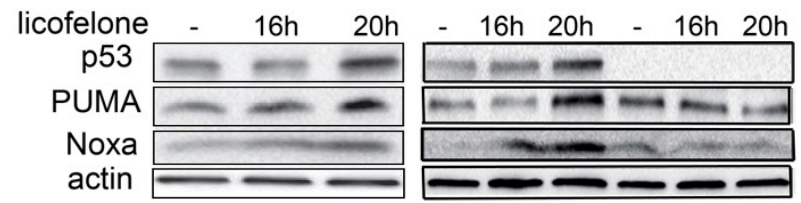

F.

RKO

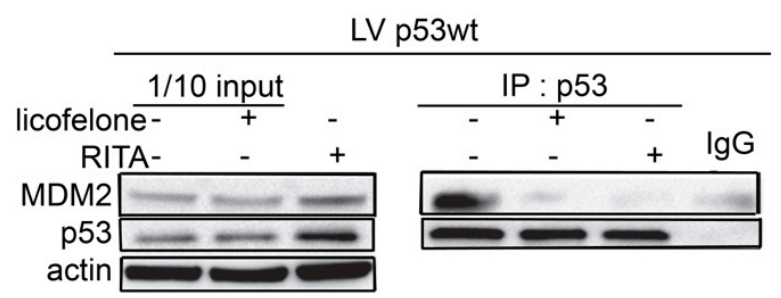

LV p53(33/37)

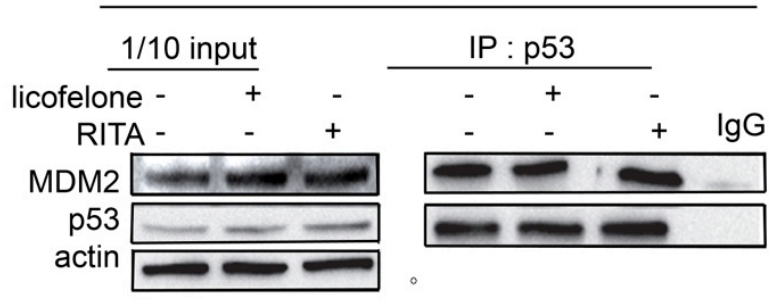

E.

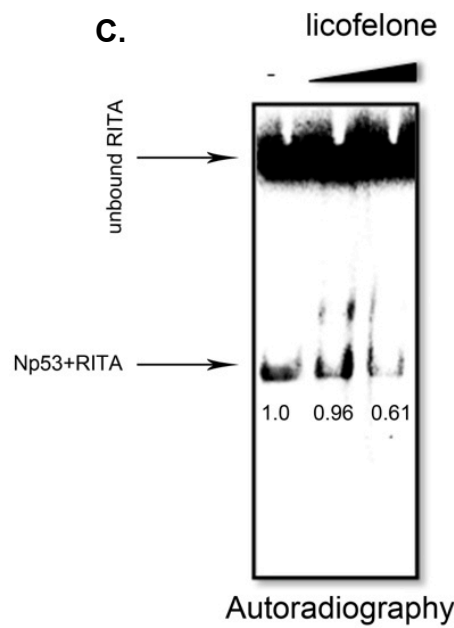

HCT 116

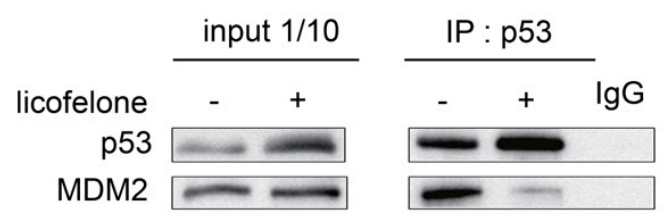

G.

RKO

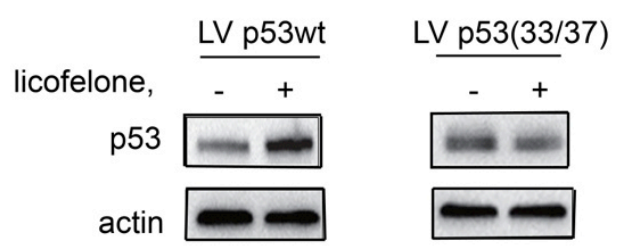

H.

HCT 116

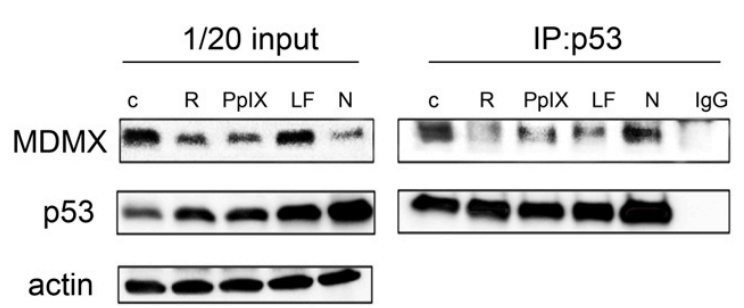

